\title{
CARACTERÍSTICAS CLÍNICAS DE PACIENTES TRATADOS CON TERAPIA ELECTROCONVULSIVA EN UN HOSPITAL PÚBLICO DE PERÚ
}

\author{
Carla Cortez-Vergara ${ }^{1,2, a}$, Lizardo Cruzado ${ }^{1,2, a}$, Ira Galia Rojas-Rojas ${ }^{1,2, a}$, \\ Miguel Sánchez-Fernández ${ }^{1,2, a}$, Guillermo Ladd-Huarachi',2,a
}

\begin{abstract}
RESUMEN
Con el objetivo de describir el perfil de uso de la terapia electroconvulsiva (TEC) en pacientes hospitalizados en el Instituto Nacional de Salud Mental "Honorio Delgado - Hideyo Noguchi" de Lima, Perú, se revisaron las historias clínicas de pacientes que recibieron TEC entre los años 2001 y 2011. Se aplicaron 419 cursos de TEC a 372 pacientes, con un total de 5439 aplicaciones; la esquizofrenia paranoide fue el diagnóstico más frecuente $(70,7 \%)$, y la indicación más usual fue la resistencia al tratamiento (80,7\%); asimismo, la respuesta clínica a la TEC fue buena en $70,1 \%$ de casos, mientras que los efectos secundarios, en general, fueron pasajeros y leves. El uso de TEC disminuyó a lo largo del periodo estudiado, pero fue muy tolerable y segura, especialmente en su versión modificada, y mostró una alta respuesta que la mantiene como un tratamiento psiquiátrico de primera línea.
\end{abstract}

Palabras clave: Terapia electroconvulsiva; Psiquiatría; Esquizofrenia paranoide (fuente: DeCS Bireme).

\section{CLINICAL CHARACTERISTICS OF PATIENTS TREATED WITH ELECTROCONVULSIVE THERAPY IN A PUBLIC HOSPITAL IN PERU}

\begin{abstract}
With the purpose of describe the profile of use of electroconvulsive therapy (ECT) on hospitalized patients at "Honorio Delgado - Hideyo Noguchi" National Institute of Mental Health in Lima, Peru, the medical records of patients receiving ECT between 2001 and 2011 were reviewed. The main findings were: four hundred and nineteen ECT courses were applied to 372 patients, with a total of 5439 applications the most common diagnosis was paranoid schizophrenia $(70.7 \%)$, the most common indication was resistance to treatment $(80.7 \%)$, also the clinical response to ECT was good in $70.1 \%$ of cases while side effects were generally transient and mild. The use of ECT decreased over the period of the study but it was tolerable and safe, especially in the modified version, and it had a high response rate so remains as a first-line psychiatric treatment.
\end{abstract}

Key words: Electroconvulsive therapy; Psychiatry; Schizophrenia, paranoid (source: MeSH NLM).

\section{INTRODUCCIÓN}

La terapia electroconvulsiva (TEC) consiste en la inducción de convulsiones tónico clónicas mediante una descarga eléctrica regulada, a nivel encefálico, previa administración de un anestésico y un relajante muscular; un curso de TEC consta de varias aplicaciones de la descarga en días diferentes. La TEC es el tratamiento que posee mayor tiempo de vigencia en la psiquiatría (1) y sigue siendo el más efectivo en varios trastornos mentales, entre ellos: depresiones melancólicas, psicóticas, con riesgo elevado de suicidio o refractarias al tratamiento farmacológico (2,3); esquizofrenia, en particular casos refractarios o en los que urge una reducción rápida de los síntomas ${ }^{(4)}$; y catatonía, específicamente si es refractaria, maligna o estuporosa ${ }^{(5)}$. Paralelamente, las indicaciones para usar TEC, cualquiera que sea el diagnóstico, incluyen: necesidad de una respuesta rápida, antecedente de resistencia al tratamiento convencional, presencia de efectos secundarios intolerables (3), preferencia del paciente ${ }^{(5)}$ o contraindicaciones para recibir medicación, por ejemplo durante la gestación ${ }^{(6)}$.

\footnotetext{
Universidad Peruana Cayetano Heredia. Lima, Perú.

Instituto Nacional de Salud Mental “Honorio Delgado - Hideyo Noguchi”. Lima, Perú.

Médico psiquiatra

Recibido: 04-05-15 Aprobado: 09-03-16
}

Citar como: Cortez-Vergara C, Cruzado L, Rojas-Rojas IG, Sánchez-Fernández M, Ladd-Huarachi G. Características clínicas de pacientes tratados con terapia electroconvulsiva en un hospital público de Perú. Rev Peru Med Exp Salud Publica. 2016;33(1):100-5. doi: 10.17843/rpmesp.2016.331.1940 
Las indicaciones de la TEC varían según la región del mundo donde se aplica, por ejemplo, la esquizofrenia es el diagnóstico más frecuente por el que se indica TEC en países en vías de desarrollo ${ }^{(7)}$. Por el contrario, en la mayoría de países europeos y en los Estados Unidos de Norteamérica, los principales diagnósticos son diversas formas de depresión ${ }^{(8-10)}$.

En Latinoamérica, las indicaciones de TEC también muestran diferencias según el país. En el Perú no existen investigaciones previas que evalúen el uso de la TEC. El objetivo del presente estudio fue describir el perfil de uso de la terapia electroconvulsiva en pacientes hospitalizados en el Instituto Nacional de Salud Mental "Honorio Delgado - Hideyo Noguchi" (INSM) entre los años 2001 y 2011.

\section{MATERIALES Y MÉTODOS}

\section{EL EQUIPO DE TEC Y LA TEC EN EL PERÚ}

Los pacientes recibieron TEC a través del equipo MECTA spECTrum 5000Q ® con pulso eléctrico ultrabreve de 0,3 milisegundos. En la TEC convencional ( $\sin$ uso de anestesia ni relajante muscular) participó el psiquiatra y médicos residentes con tres enfermeros. En la TEC modificada se añadió la presencia de un médico anestesiólogo.

La TEC fue introducida en el Perú en enero de 1943 por Honorio Delgado ${ }^{(11)}$. En la actualidad, no existe una asignatura formal dedicada a la enseñanza de TEC a los médicos residentes de psiquiatría en Perú (12).

\section{DESCRIPCIÓN DE LA MUESTRA}

Se incluyeron a todos los pacientes hospitalizados en el INSM desde el año 2001 hasta el año 2011. El INSM es el principal hospital psiquiátrico del Perú, se ubica en la capital, Lima, que cuenta con 8 millones y medio de habitantes y atiende a la tercera parte de dicha población (mayoritariamente urbano-marginal). El INSM es uno de los diez hospitales estatales en el Perú que ofrece TEC (para un total de 31 millones de habitantes) y cuenta con aproximadamente 80 camas de internamiento. Durante el periodo de estudio, 2652 pacientes fueron hospitalizados y $372(14,0 \%)$ de ellos recibieron TEC (el porcentaje es $15,8 \%$ si consideramos los 419 cursos de TEC).

\section{PROCEDIMIENTOS}

Se recogieron los datos a partir de la revisión de historias clínicas de los pacientes hospitalizados que recibieron TEC desde el año 2001 al 2011, previa aprobación del comité de ética del INSM. Los datos recolectados fueron la edad, sexo, fecha de aplicación de la TEC, diagnóstico principal de acuerdo con la Clasificación Internacional de Enfermedades (CIE-10), uso previo de TEC (dentro del periodo de estudio), indicación principal, número de aplicaciones recibidas, uso de atropina, anestesia y relajantes musculares, carga administrada, consentimiento informado, resultado del electrocardiograma previo, medicación concomitante y efectos secundarios. Debido a que no se contó con parámetros estandarizados del grado de mejoría en respuesta a la TEC, este indicador fue categorizado clínicamente en: buena respuesta (gran mejoría, remisión completa o casi completa de todos los síntomas); respuesta moderada (mejoría o remisión parcial de los síntomas); sin respuesta (sin cambios o empeoramiento), y respuesta no evaluable, según lo descrito en la historia clínica. Asimismo, se determinó el número total de cursos de TEC por año.

Se realizaron análisis estadísticos univariados y bivariados. Se utilizó la prueba de chi cuadrado de Pearson para valorar la asociación de variables cualitativas. El procesamiento de los datos se efectuó con el programa SPSS v22.0.

\section{RESULTADOS}

Entre los años 2001 al 2011 se aplicaron un total de 419 cursos de TEC a 372 pacientes. El número total de aplicaciones fue 5439 . El rango de edades fue de 16 a 72 años, con un promedio de $30 \pm 10,6$ años. El grupo etario más frecuente fue el de 20 a 29 años en ambos sexos; el $50 \%$ de los pacientes tuvo 27 años o menos. El $59,7 \%$ de cursos fue realizado en varones. El mayor porcentaje de cursos $(18 \%)$ se llevó a cabo en el año 2002 y el menor en el año 2011 (3,9\%) (Figura 1).

El diagnóstico más frecuente por el cual se indicó TEC fue esquizofrenia paranoide $(70,7 \%)$, seguido de trastorno bipolar (episodio actual maniaco) y esquizofrenia catatónica (6,7 y $6,2 \%$ respectivamente) (Tabla 1).

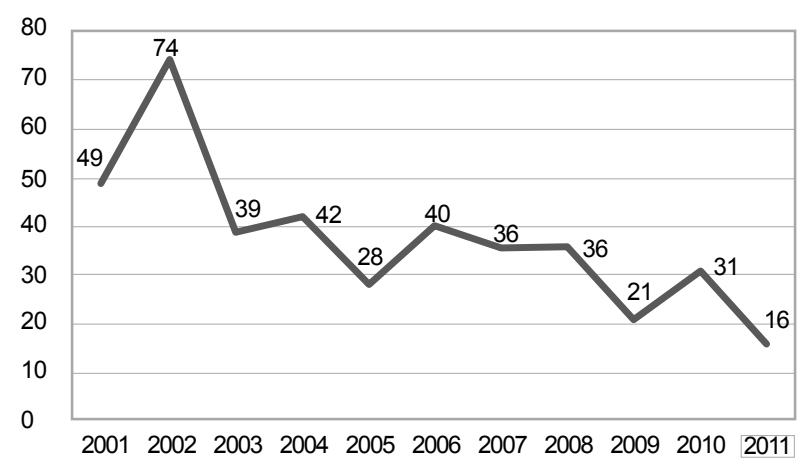

Figura 1. Tendencia de uso de TEC a lo largo del periodo de estudio 
Tabla 1. Pacientes hospitalizados que recibieron TEC, según diagnóstico psiquiátrico

\begin{tabular}{lrr}
\hline Diagnóstico & $\mathbf{N = 3 7 2}$ & $\mathbf{( \% )}$ \\
\hline Trastorno psicótico (N=324) & & \\
\hline Esquizofrenia paranoide & 263 & $(70,7)$ \\
\hline Esquizofrenia catatónica & 23 & $(6,2)$ \\
\hline Esquizofrenia desorganizada & 15 & $(4,0)$ \\
\hline Esquizofrenia indiferenciada & 11 & $(3,0)$ \\
\hline Trastorno esquizoafectivo & 6 & $(1,6)$ \\
\hline Trastorno esquizofreniforme & 3 & $(0,8)$ \\
\hline Esquizofrenia no especificada & 3 & $(0,8)$ \\
\hline Total & 324 & $(87,1)$ \\
\hline Trastornos depresivos (N=23) & & \\
$\quad \begin{array}{l}\text { Episodio depresivo con síntomas } \\
\text { psicóticos }\end{array}$ & 22 & $(5,9)$ \\
\hline $\begin{array}{l}\text { Episodio depresivo sin síntomas } \\
\text { psicóticos }\end{array}$ & 1 & $(0,3)$ \\
\hline Total & 23 & $(6,2)$ \\
\hline Trastorno bipolar (N=25) & & \\
\hline Episodio actual maniaco & 25 & $(6,7)$ \\
\hline
\end{tabular}

Las indicaciones de uso más frecuentes fueron resistencia al tratamiento $(80,7 \%)$, seguida de la ideación suicida o autolesiva persistente $(7,4 \%)$ y la intolerancia a efectos secundarios de algunos psicofármacos $(6,2 \%)$. Ochenta cursos contaron con más de una indicación (Tabla 2).

Tabla 2. Indicaciones para uso de TEC durante el periodo de estudio

\begin{tabular}{lcc}
\hline Indicación de la TEC & N & $\left(\%^{*}\right)$ \\
\hline Refractariedad al tratamiento & 338 & $(80,7)$ \\
\hline $\begin{array}{l}\text { Ideación suicida / autolesiva } \\
\text { persistente }\end{array}$ & 31 & $(7,4)$ \\
$\begin{array}{l}\text { Efectos secundarios intolerables de } \\
\text { la medicación }\end{array}$ & 26 & $(6,2)$ \\
\hline $\begin{array}{l}\text { Síntomas catatónicos } \\
\text { (rechazo de alimentos) }\end{array}$ & 24 & $(5,7)$ \\
\hline $\begin{array}{l}\text { Agresividad o desorganización } \\
\text { conductual severas }\end{array}$ & 18 & $(4,3)$ \\
\hline $\begin{array}{l}\text { Falta de dinero para compra de } \\
\text { medicación }\end{array}$ & 18 & $(4,3)$ \\
\hline Antecedente de buena respuesta & 16 & $(3,8)$ \\
\hline $\begin{array}{l}\text { Severidad de la sintomatología } \\
\text { Necesidad de recuperación rápida }\end{array}$ & 11 & $(2,6)$ \\
\hline $\begin{array}{l}\text { Rechazo del tratamiento oral } \\
\text { Antecedente de síndrome } \\
\text { neuroléptico maligno }\end{array}$ & 8 & $(1,9)$ \\
\hline $\begin{array}{l}\text { Gestación } \\
\text { No especificado }\end{array}$ & 5 & $(1,9)$ \\
\hline
\end{tabular}

* Respecto a un total de 419 cursos. Un curso de TEC pudo tener más de dos indicaciones
El número mínimo de aplicaciones por curso de TEC fue uno (se suspendió en un paciente que sufrió fractura en una vértebra dorsal lesionada previamente). El máximo número de aplicaciones fue 44 y el promedio fue $14 \pm 6$. El $52,1 \%$ de pacientes recibió de 6 a 12 aplicaciones por curso de TEC. De los pacientes que recibieron más de un curso de TEC durante el periodo de estudio, se encontró que, en el $13,2 \%$ de los casos, los pacientes habían recibido TEC anteriormente.

Se utilizó atropina en el $93,5 \%$ de cursos, en el $44,2 \%$ se utilizaron anestésicos y en el $32,7 \%$ relajantes musculares. En el caso de los anestésicos, se observó incremento considerable de su uso a partir del año 2006 (al 100\%) y en el caso de los relajantes musculares, su uso fue incrementándose progresivamente hacia el año 2006 y 2007 (32 y 43,3\% respectivamente), alcanzando 100\% en el año 2009. Los anestésicos más usados fueron propofol (93 casos), pentotal (19 casos) y ketamina (9 casos). En 98 de los casos se usó succinilcolina como relajante muscular. La carga eléctrica promedio administrada fue 115,7 \pm 46,6 miliamperios, siendo la mínima 48 y la máxima 294 miliamperios. La aplicación de los electrodos fue siempre bitemporal. En cuanto al consentimiento informado para la TEC, en $73,2 \%$ de casos firmaron el consentimiento los familiares, en $2,2 \%$ el paciente, en el $1,7 \%$ ambos y en el resto no se pudo determinar.

El electrocardiograma previo al procedimiento fue informado en el 89,6\% como normal, en 9,6\% anormal, pero sin importancia clínica (taquicardias o bradicardias sinusales) y en el $0,8 \%$ (tres casos) fue patológico, aunque no contraindicó la TEC. Con relación a la medicación concomitante, los pacientes recibieron antipsicóticos en el $94,3 \%$ de casos; hipnóticos en el $27,3 \%$ y otros medicamentos en el $38,5 \%$ (de estos, la mayoría recibió anticolinérgicos como biperideno). Solo $5,7 \%$ de pacientes recibieron TEC sin ningún psicofármaco concomitante.

La respuesta clínica a la TEC fue catalogada como buena en $70,1 \%$ de los casos, parcial en $26 \%$ y sin respuesta en el 3,1\% (doce pacientes). En estos últimos casos se relacionó la ausencia de respuesta clínica al hecho de haberse presentado previamente una evolución tórpida y efectos secundarios a la TEC como síndrome confusional.

Finalmente, se verificaron efectos secundarios de la TEC en el $46,9 \%$ de cursos. La confusión y la amnesia fueron los efectos secundarios más frecuentes. Hubo siete casos de complicaciones traumatológicas (luxaciones de hombro, fractura subcapital de húmero, fractura de vértebra dorsal -en paciente con lesión ósea previa-, fisura de omóplato y luxación de piezas dentarias) pero todas ellas sucedieron con TEC convencional (Tabla 3). 
Tabla 3. Principales efectos secundarios de la TEC durante el periodo de estudio

\begin{tabular}{lcc}
\hline Efectos secundarios de la TEC & $\mathbf{N}$ & $\left(\%^{*}\right)$ \\
\hline Confusión & 73 & $(17,4)$ \\
\hline Alteraciones de memoria & 45 & $(10,7)$ \\
\hline Cefalea & 21 & $(5,0)$ \\
\hline Mialgias o contracturas musculares & 16 & $(3,8)$ \\
\hline $\begin{array}{l}\text { Agitación o ansiedad previa al } \\
\text { procedimiento }\end{array}$ & 9 & $(2,1)$ \\
\hline $\begin{array}{l}\text { Traumatológicos } \\
\text { (luxación, fracturas) }\end{array}$ & 7 & $(1,7)$ \\
\hline Apnea prolongada & 5 & $(1,2)$ \\
\hline Hipertensión arterial & 5 & $(1,2)$ \\
\hline Somnolencia & 5 & $(1,2)$ \\
\hline $\begin{array}{l}\text { Otros/ as ** } \\
\text { Total }\end{array}$ & 11 & $(2,6)$ \\
\hline * Respecto a un total de 419 cursos de TEC \\
** Incluye: convulsiones prolongadas (3), rash dérmico (3), delirium (2), \\
shock neurogénico (1), hemorragia digestiva alta (1), gingivorragia (1)
\end{tabular}

No hubo diferencias significativas en cuanto a respuesta clínica, indicaciones de uso, número de sesiones, ni en el resto de efectos secundarios entre TEC convencional y TEC modificada.

\section{DISCUSIÓN}

La mayoría de los pacientes usuarios de TEC en nuestro estudio fueron adultos jóvenes. Estos datos ubican a nuestra muestra por debajo de otras donde el promedio etario es por encima de los 40 años de edad $(10,13,14)$ como lo reportado en la mayoría de países europeos y en los Estados Unidos. Una edad promedio menor fue hallada en Colombia y Brasil, promedios de edad similares fueron reportados en muestras de México, Sudáfrica, Rusia, Turquía, India y Arabia ${ }^{(14)}$.

Los varones constituyeron la mayoría de pacientes que recibieron TEC en nuestro estudio. Esto contrasta con referencias de Brasil, Estados Unidos y Australia, donde la mayoría de pacientes suelen ser de sexo femenino ${ }^{(13,14)}$. Son excepciones países como la India y Uruguay donde la proporción es mayor en el sexo masculino ${ }^{(14,15)}$. La predominancia masculina y juvenil de la muestra puede explicarse por el mayor número de pacientes con esquizofrenia, pues los varones inician a edad más temprana su enfermedad y sufren mayor número de internamientos.

El porcentaje de pacientes que recibieron TEC $(15,8 \%)$ fue menor al de países del África, donde entre 21 y $28 \%$ de pacientes hospitalizados son tributarios de TEC, o en Asia, donde dicho porcentaje puede llegar hasta el $26 \%$, dependiendo de los estudios, pero bastante por encima de Estados Unidos donde solo $1,3 \%$ de pacientes reciben TEC, y de los países europeos donde los rangos van desde $0,6 \%$ (Hungría) hasta $14 \%$ (Turquía) ${ }^{(14)}$. En Latinoamérica, nuestros resultados están por debajo de Chile $(27,5 \%)$ o Brasil $(33,2 \%){ }^{(10)}$, pero son similares a lo registrado en Uruguay $(15 \%){ }^{(15)}$.

En cuanto a los diagnósticos tributarios de indicación de TEC, el primer lugar fue ocupado por la esquizofrenia y trastornos relacionados, con $87,1 \%$; mientras que los problemas depresivos fueron el $6,2 \%$. Esta proporción es semejante a la que se aprecia en países asiáticos y africanos, por ejemplo, en la India se reporta que el $75 \%$ de pacientes tratados con TEC son esquizofrénicos, en la región Asia-Pacífico lo son el $68 \%$, en África tal proporción oscila entre 60 y $83 \%$, e igualmente en Asia continental, con excepción de Arabia, Pakistán y Hong Kong, la predominancia corresponde a los trastornos esquizofrénicos como indicación para TEC. Nuestro $87,1 \%$ es superior, incluso, a la cifra registrada en Tailandia (74\%). Análogamente, nuestro hallazgo es contrapuesto a los datos prevalentes en Europa (con excepción de Hungría y Turquía) y Estados Unidos de América, donde los trastornos depresivos en sus diversos subtipos son los diagnósticos que sustentan con mayor frecuencia la indicación de TEC ${ }^{(14)}$.

Sin embargo, dentro del ámbito sudamericano hay divergencias en los diagnósticos más frecuentes: en un estudio colombiano primó la depresión en todos sus tipos

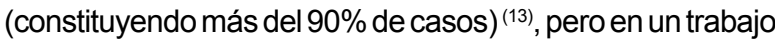
brasileño la esquizofrenia y trastornos relacionados fueron mayoría, con $55,1 \%{ }^{(10)}$. Probablemente esto fue influido por la procedencia de la muestra: el trabajo colombiano fue de un centro privado y el brasileño de un hospital público. Por otra parte, en México ${ }^{(16)}$ se encontró que los trastornos depresivos indicaron $50 \%$ de las veces la TEC, mientras que la esquizofrenia y otras psicosis no afectivas justificaron solamente $29 \%$ de los casos. En Uruguay, en cambio, encontramos una cifra mayor para esquizofrenia $(55 \%)$, y si añadimos trastorno esquizoafectivo, trastorno delusional y psicosis agudas, la cifra llega a $66,1 \%$ mientras que la suma de depresiones uni y bipolares alcanzaron solo el $12,9 \%{ }^{(15)}$. Tanto el estudio mexicano como el uruguayo se efectuaron en hospitales públicos.

En el presente estudio, los pacientes recibieron un promedio de $14 \pm 6$ aplicaciones de TEC, cifra mayor al promedio de países europeos y de Estados Unidos (14), casi el $50 \%$ de nuestros pacientes recibió más de trece aplicaciones. En un estudio realizado en Colombia el promedio fue 7,37 aplicaciones por paciente ${ }^{(13)} y$ en Brasil 
se halló un promedio de ocho aplicaciones por paciente ${ }^{(10)}$. en México el promedio fue de $9,2 \pm 2,5^{(16)}$ y en Uruguay $9,9 \pm 2,5^{(15)}$. El elevado promedio en nuestro trabajo es explicable por ser la esquizofrenia refractaria la principal causa de uso de TEC en nuestro centro, cuadro que usualmente requiere mayor número de aplicaciones que otros diagnósticos ${ }^{(17)}$.

La principal indicación de TEC fue resistencia al tratamiento, que coincide con la mayor frecuencia de esquizofrenia como diagnóstico sustentador de la TEC. Debemos anotar que en los años del estudio (20012011), en Perú no había cobertura gratuita de salud para la mayoría de la población, por lo que la imposibilidad de acceso a los antipsicóticos atípicos, obviamente más caros, podría haber fomentado la estipulación de casos de esquizofrenia como "refractarios" y propiciado la indicación de TEC en un entorno donde muchos psicofármacos eran de difícil acceso y no quedaba otra opción que el uso de TEC como alternativa de bajo coste, probada eficacia y resultado rápido. Aunque el uso de TEC en esquizofrenia ha sido discutido por algunos autores, las guías de la Asociación Mundial de Psiquiatría -entre otras- sustentan su empleo y, por tanto, los hallazgos de nuestro trabajo ${ }^{(17)}$.

Las otras indicaciones para uso de TEC, en nuestro estudio, fueron, en comparación, minoritarias: aunque la catatonía es más frecuente en países subdesarrollados, ello no se muestra en nuestros resultados, así como tampoco su uso en riesgo suicida elevado. Hipotéticamente, puede existir una falla en reconocer a la catatonía entre nosotros y puede también existir una proclividad -en la población y los profesionales- a asociar la TEC con casos severos de esquizofrenia y refractarios, lo que reforzaría su uso para este fin ${ }^{(18)}$. No existe uniformidad en los estudios consultados acerca de la diferenciación entre diagnóstico subyacente al uso de TEC y la indicación que suscita dicho uso, en muchos de ellos simplemente se amalgaman ambas características y eso dificulta las comparaciones ad hoc.

Como se ha señalado, a partir del año 2006, en el Perú se estipuló la obligatoriedad del uso de TEC modificada. Desde entonces, todos los procedimientos debieron efectuarse usando anestésico más relajante muscular, sin embargo, dado que no existía cobertura de seguro de salud y los familiares debieron cubrir los costos del servicio, en muchas ocasiones se utilizó incompletamente el esquema de TEC modificada, lo que se fue subsanando con el paso del tiempo. En la actualidad, todos los procedimientos de TEC en nuestro centro se efectúan con empleo de anestesia y relajante muscular.

Con relación a los efectos secundarios, el presente estudio encontró que los más frecuentes fueron la confusión y la amnesia, en porcentajes similares a lo descrito en otros estudios ${ }^{(10,14)}$, y fueron leves y de breve duración, aunque también se presentaron efectos secundarios de mayor seriedad como apneas prolongadas, hipertensión arterial y complicaciones traumatológicas, aunque en escasa cuantía y no se registró ningún fallecimiento. Debe resaltarse que las siete complicaciones traumatológicas sucedieron con la TEC no modificada.

La respuesta a la TEC se consideró buena en $70,1 \%$ de los casos, similar al porcentaje hallado en muestras con predominio de personas con esquizofrenia pues en las que predominan los depresivos, puede hallarse hasta $90 \%$ de efectividad ${ }^{(3)}$.

En nuestra muestra hubo uso de antipsicóticos en $94,3 \%$ de los casos, lo que se explica por la mayoría de casos de esquizofrenia. Se ha verificado mejor evolución clínica si se suman psicofármacos a la TEC en esta indicación ${ }^{(17)}$.

Otro aspecto que considerar es la tendencia a la disminución del uso de TEC. Es posible que a lo largo de los años, la creciente disponibilidad de antipsicóticos haya devenido en un menor uso de TEC, tal como observamos en el presente estudio. No obstante, aún existe una fuerte estigmatización con relación a su uso, debido a la imagen que se le ha dado en distintos medios ${ }^{(18)}$. Esto podría ser contraproducente ya que, a pesar de las críticas, sigue siendo un tratamiento sumamente efectivo y seguro cuando se realiza en las condiciones apropiadas ${ }^{(19,20)}$. La carencia de formación profesional específica en TEC, como señalamos antes, también puede haber influido en esta disminución de uso de la TEC.

En cuanto a las limitaciones del presente trabajo, señalamos que la valoración de la respuesta a la TEC se realizó por la apreciación clínica registrada en la historia clínica, la cual es parcialmente subjetiva; por esta razón, sugerimos la necesidad de evaluar la respuesta con instrumentos adicionales apropiados. Asimismo, el que nuestra muestra provenga de una institución especializada en psiquiatría podría aportar algunos sesgos.

En conclusión, en el INSM, la TEC se aplica predominantemente a varones jóvenes con diagnóstico de esquizofrenia, a causa mayormente de resistencia al tratamiento, aunque su uso ha ido disminuyendo a lo largo de los años estudiados. En general, el uso de TEC es bien tolerado y presenta una elevada frecuencia de respuesta. Es urgente despejar el estigma social que acompaña a esta técnica, dignificando un tratamiento avalado totalmente por la evidencia científica ${ }^{(20)}$. 
Agradecimientos: a la Mag. Ysela Agüero Palacios por su asesoría estadística; al personal de la Oficina de Estadística e Informática del Instituto Nacional de Salud Mental "Honorio Delgado - Hideyo Noguchi".

Contribuciones de autoría: CCV y LC diseñaron el estudio. CCV, GRR y MSF recolectaron e interpretaron los datos. CCV y
LC redactaron el artículo. GLH efectuó la revisión crítica y todos los autores aprobaron la versión final.

Fuente de financiamiento: autofinanciado.

Conflictos de interés: los autores declaran que no tienen conflictos de interés.

\section{REFERENCIAS BIBLIOGRÁFICAS}

1. Lebensonn $Z$. The history of electroconvulsive therapy in the United States and its place in American psychiatry: a personal memoir. Compr Psychiatry. 1999; 40(3): 173-81. doi:10.1016/S0010440X(99)90000-7

2. Pagnin D, de Queiroz V, Pini S, Cassano GB. Efficacy of ECT in depression: a meta-analytic review. J ECT. 2004; 20(1):13-20.

3. Husain MM, Lisanby SH, Kay J. Brain Stimulation in Psychiatry. En: Tasman A, Kay J, Lieberman JA, First MB, Riba MB, editors. Psychiatry. 4a ed. Chichester: John Wiley \& Sons, Ltd; 2015. pp. 2291-2310.

4. Tharyan P, Adams C. Electroconvulsive therapy for schizophrenia. Cochrane Database Syst Rev. 2005;18(2): CD000076. doi: 10.1002/14651858. CD000076.pub2

5. Raveendranathan D, Narayanaswamy JC, Reddi S. Response rate of catatonia to electroconvulsive therapy and its clinical correlates.Eur Arch Psychiatry Clin Neurosci. 2012;262(5):425-30. doi: 10.1007/s00406-011-0285-4

6. American Psychiatric Association. Committee on Electroconvulsive Therapy. The practice of electroconvulsive therapy: recommendations for treatment, training and privileging: a task force report of the American Psychiatric Association. Washington DC: American Psychiatric Association; 2001.

7. Agarwal AK, Andrade C, Reddy MV. The practice of ECT in India: Issues Relating to the Administration of ECT. Indian J Psychiat. 1992;34(4): 285-97.

8. Schweder LJ, Lydersen S, Wahlund B, Bergsholm P, Linaker O.
Electroconvulsive therapy in Norway: rates of use, clinical characteristics, diagnoses, and attitude.J ECT. 2011; 27(4):292-5. doi: 10.1097/ YCT.0b013e318208e24b

9. Saatcioglu O, Tomruk N. Practice of electroconvulsive therapy at the research and training hospital in Turkey. Soc Psychiatry Psychiatr Epidemiol. 2008;43(8):673-7. doi: $10.1007 / \mathrm{s} 00127-008-0351-\mathrm{z}$

10. Pastore D, Marchetti L, Nardi A, Gonçalves A. O uso da eletroconvulsoterapia no Instituto de Psiquiatria da Universidade Federal do Rio de Janeiro no período de 2005 a 2007. Rev psiquiatria Rio Gd Sul. 2008;30(3):175-81. doi: 10.1590/ S0101-81082008000400006

11. Delgado C. Tratamiento de las enfermedades mentales por electroshock. Rev Neuropsiquiatr. 1943; 6: 263-316.

12. Ocampo-Zegarra JC, Cortez-Vergara C, Alva-Huerta M, Rojas-Rojas G. Encuesta a médicos residentes de psiquiatría sobre la calidad de su formación como especialistas. Rev Neuropsiquiatr. 2013; 76: 109-119. doi: $10.20453 /$ rnp.v76i2.1193

13. Ocampo MV, Ramírez CI, Franco JG, Gómez LM, Cardona G, Restrepo C. Características clínicas de 276 pacientes tratados con terapia electroconvulsiva en una clínica universitaria de Medellín, Colombia. Rev Colomb Psiquiat. 2012;41(2):357-70. doi: 10.1016/S0034-7450(14)60010-X

14. Leiknes KA, Jarosh-von Schweder L, Høie B. Contemporary use and practice of electroconvulsive therapy worldwide. Brain Behav. 2012; 2: 283345. doi: 10.1002/brb3.37

15. Wschebor M, López-Rega G, Santos V, Brescia S, Romano S. Estudio de los pacientes que recibieron ECT en el Hospital Vilardebó en el año 2006. Rev Psiquiatr Urug. 2009;73(2):131-42.

16. Almeida L, Flores M, Palacios L, Garnica R, de la Peña F, Juárez J, Cortés J, Heinze G. Terapia electroconvulsiva: experiencia en el Instituto Mexicano de Psiquiatría 1995-1998. Salud Mental. 1999; 22 (4): 33-40.

17. Salleh MA, Papakostas I, Zervas I, Christodoulou G. Eletroconvulsoterapia: critérios e recomendações da Associação Mundial de Psiquiatria. Rev psiquiatr clín. 2006;33(5):262-7. doi: 10.1590/ S0101-60832006000500006

18. Payne NA, Prudic J. Electroconvulsive Therapy Part II: A Biopsychosocial Perspective. J Psychiatr Pract. 2009;15(5)369-90. doi: 10.1097/01. pra.0000361278.73092.85

19. Fink M. Electroconvulsive therapy resurrected: its successes and promises after 75 years. Can J Psychiatry. 2011;56(1):3-4.

20. Bernardo M, Urretavizcaya M. Dignificando una terapia electroconvulsiva basada en la evidencia. Rev Psiquiatr Salud Ment. 2015;8(2):51-54. doi: 10.1016/j. rpsm.2015.01.002

Correspondencia: Carla Cortez-Vergara

Dirección: Instituto Nacional de Salud Mental "Honorio Delgado - Hideyo Noguchi”. Jr. Eloy Espinoza 709. Urb. Palao. San Martin de Porres. Lima, Perú.

Teléfono: (511) 971134800

Correo electrónico:cortez_vergara@yahoo.com 